\title{
Fast Retransmission of Real-Time Traffic in HIPERLAN/2 Systems
}

\author{
José A. Afonso and Joaquim E. Neves \\ Department of Industrial Electronics \\ University of Minho, Campus de Azurém \\ 4800-058 Guimarães, Portugal \\ \{jose.afonso,jeneves\}@dei.uminho.pt
}

\begin{abstract}
Automatic repeat request (ARQ) schemes are effective to recover non-real-time data corrupted by channel errors, but their use with real-time traffic is seldom considered because packet retransmissions tend to increase the delay beyond the deadline. However, the high-speed of modern wireless local area networks, together with their inherent low propagation times, favors the use of $A R Q$ schemes with real-time traffic. This paper proposes a fast retransmission scheduling mechanism for reliable transport of realtime traffic in HIPERLAN/2 systems, as well as an adaptive rate scheme designed to reduce the delay introduced by retransmissions while keeping the same level of network efficiency. The achieved performance is evaluated in the scenario of a distributed data acquisition and control system.
\end{abstract}

\section{Introduction}

Wireless networks present many advantages over their wired counterparts derived from the freedom from cables, such as the reduction of costs with infrastructure, quicker installation and increased mobility. On the other side, these networks are subject to higher and more variable channel error rates.

Several scheduling mechanisms have been proposed in the literature for use in wired networks, such as the Weighted Fair Queueing (WFQ), suitable for handling asynchronous traffic, and the Earliest Due Date (EDD), for real-time traffic [1]. However, they are not appropriate for wireless networks because of the assumption of an error free channel. Moreover, the access point does not know when a packet arrives to the queue of a remote station, so it cannot rely on the packet tagging approach used by conventional mechanisms. A practical implementation of a scheduling mechanism should address these issues, as well as some characteristics of the wireless network, such as its medium access control (MAC) and error control (EC) functions.

Many papers that deal with scheduling in wireless networks and consider the channel errors have been published recently [2] [3], but their main concern is the fair sharing of the available bandwidth among asynchronous traffic flows. In this sense, the emphasis is mostly given to the throughput instead of the delay requirements of the flows.

Regarding to HIPERLAN/2 systems, $\mathrm{Li}$ et al. presented a MAC performance analysis in [4], but it considers only one connection. Kadelka et al. [5] presented results for a scenario with multiple terminals; however, the error control mechanism was neglected. Doufexi et al. [6] presented similar results concerning the MAC layer performance. These papers make simplifying assumptions in relation to the scheduling of data, resource request (RR) and ARQ messages. Besides, the results presented for the MAC layer are restricted to the aggregate throughput of all connections.

In [7], we proposed scheduling mechanisms designed to provide the efficient transport of real-time traffic subject to channel errors in IEEE 802.11 networks. In this paper, we make a similar proposal concerning HIPERLAN/2 systems. The achieved results are provided per individual connection and address particularly the delay performance for time critical applications.

Next section presents an overview of the HIPERLAN/2 standard; Section 3 describes the proposed scheduling mechanisms, while section 4 presents simulation results obtained with these mechanisms. Finally, the conclusions are presented on section 5 . 


\section{Overview of HIPERLAN/2}

HIPERLAN/2 [8] is a standard for high-speed radio communication proposed by ETSI (European Telecommunications Standard Institute) to connect portable devices to broadband networks based on ATM, IP and other technologies. It operates in the 5 $\mathrm{GHz}$ band using Orthogonal Frequency Division Multiplexing (OFDM) and provides an extensive QoS support. Seven transmission modes are defined, ranging from $6 \mathrm{Mbit} / \mathrm{s}$ to $54 \mathrm{Mbit} / \mathrm{s}$.

The MAC protocol of HIPERLAN/2 [9] is a demand based dynamic TDMA/TDD mechanism which works under the coordination of the access point (AP). The basic MAC frame format is displayed on Figure 1. The MAC frame duration is fixed (2 $\mathrm{ms})$, but the duration of each phase depends on the demand.

\begin{tabular}{|c|c|c|c|c|c|}
\hline \multicolumn{3}{|c|}{ Broadcast Phase } & Downlink Phase & Uplink Phase & $\begin{array}{c}\text { Random Access } \\
\text { Phase }\end{array}$ \\
\hline ВCH & FCH & $\begin{array}{l}\mathbf{A} \\
\mathbf{C} \\
\mathbf{H}\end{array}$ & & & RCHs \\
\hline
\end{tabular}

Figure 1. HIPERLAN/2 MAC frame structure

In order to schedule the transmissions over the wireless channel, the access point (AP) needs to know the state of its own buffers and the buffers of the mobile terminals (MT). The terminals report their buffer states using Resource Request (RR) messages, either on the uplink phase or on the random access phase. The MTs can also negotiate with the AP for a periodic allocation of resources by means of the Fixed Capacity Agreement (FCA) procedure. The resource allocation information is conveyed by Resource Grant (RG) messages inserted on the Frame control CHannel (FCH).

The Broadcast control CHannel $(\mathrm{BCH})$ is used by the AP to broadcast basic cell information, while the Access feedback CHannel (ACH) provides feedback on the random access attempts made by the MTs on the previous frame. The downlink and uplink phases are composed of PDU trains, which consist of a sequence of transport channels. The Long transport CHannel (LCH) (54 bytes, with 48 bytes of payload) is primarily used to carry data messages, while the Short transport CHannel (SCH) (9 bytes) conveys control messages, like RR and ARQ messages. Finally, the random access phase is composed of a number of Random access CHannel (RCH) slots.

$\mathrm{BCH}, \mathrm{FCH}, \mathrm{ACH}$ and $\mathrm{RCH}$ PDUs are transmitted at the minimum rate $(6 \mathrm{Mbit} / \mathrm{s})$. The transmission mode used with LCH and SCH PDUs can vary.

On top of the DLC layer is the Convergence Layer (CL), which provides functions as segmentation and reassembly (SAR) and header translation to the higher layers. Two types of CL are currently defined: a cell based one, which is used with ATM networks, and a packet based one, used with IP networks.

\section{Proposed Mechanisms}

\subsection{Typical Retransmission Process}

Before we describe the proposed scheduling mechanisms, we present, in Figure 2, an example of a typical packet retransmission process in HIPERLAN/2. At frame $x, 3 \mathrm{LCH}$ channels (requested previously by the MT) are used to carry data packets in the uplink phase (UL) for a particular connection, but packets $i$ and $i+2$ are received with errors by the AP.

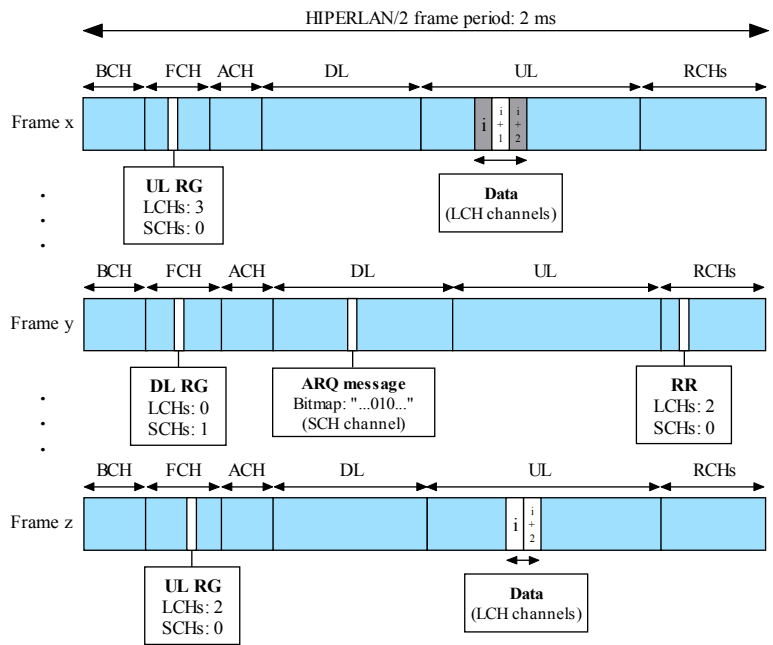

Figure 2. Typical packet retransmission process

At frame $y$ the AP sends an ARQ message to the MT which performs the positive acknowledgement of packet $i+1$ and the negative acknowledgement of packets $i$ and $i+2$. In the same frame (or later), the MT requests $2 \mathrm{LCH}$ channels during the random access phase, using a RR message, to be able to make the retransmission the corrupted packets.

As the RR message can be lost by collision, further request attempts may have to be made in the following frames. After the AP successfully receives the RR message, it allocates $2 \mathrm{LCH}$ channels at the frame $z$, where the lost packets are finally retransmitted. If a retransmission fails the whole process has to be repeated again.

Each retransmission attempt increases the packet delay by a minimum of 2 frames (when $y=x+1$ and $z=y+1$ ). This additional delay can in fact be much larger, depending on the delay between the reception of 
the corrupted packet and the transmission of the ARQ message by the AP, as well as the number of collisions suffered by the RR message (which is influenced by the traffic load on the random access phase).

\subsection{Fast Retransmission Mechanism}

Figure 3 presents an example of the operation of the fast retransmission scheduling mechanism. Frame $x$ is identical to the one used on Figure 2.

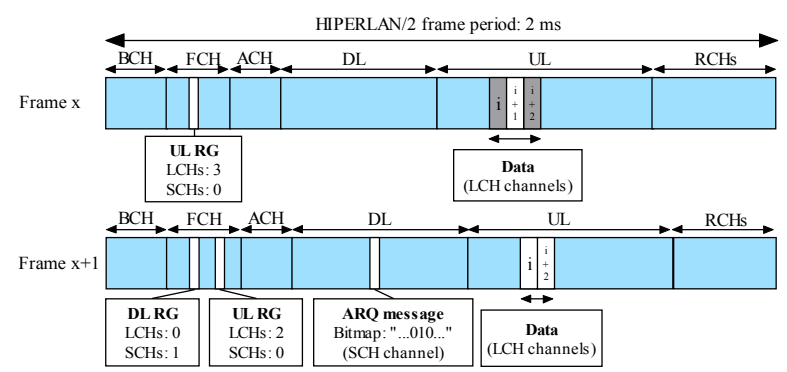

\section{Figure 3. Fast retransmission scheduling mechanism}

According to the proposed mechanism, when the AP doesn't receive a valid packet at a granted $\mathrm{LCH}$ channel, it schedules immediately the transmission of an ARQ message in the following frame. In addition, the AP grants the additional resources necessary for the retransmission of the corrupted packets automatically.

Thus, at the next frame $(x+1)$, the AP sends an ARQ message which allows the identification of the corrupted packets by the MT. As the uplink phase is located after the downlink phase on the HIPERLAN/2 frame, the AP can grant the $2 \mathrm{LCH}$ channels required for retransmission in the same frame.

This mechanism enables the reduction of the retransmission delay to just one frame, instead of two or more frames as in the typical process. In addition, the mechanism reduces the contention during the random access phase by avoiding the transmission of RR messages.

\subsection{Adaptive Rate Scheme}

We also propose a simple rate adaptation scheme which aims to reduce the delay generated by the retransmission process without compromising the efficiency. The rationale is that a lower transmission mode is more robust against channel errors [5] allowing the reduction of the number of retransmissions and consequently, of the delay variation. However, the lower mode is inefficient when the channel conditions are good.
According with this scheme, the original transmission of each packet uses the nominal transmission rate. Whenever a packet is corrupted, its retransmissions are made at the lowest transmission mode, in order to increase the probability of a successful reception.

\section{Simulation Model and Results}

In order to evaluate the performance of the proposed mechanisms, we developed a detailed model of the HIPERLAN/2 network based on the standard. This model was implemented using OMNET++ [10], an open-source discrete event simulation tool. Results were obtained from steady-state simulations.

The evaluation scenario consists of 30 terminals which transmit data to an access point. Each terminal generates CBR (Constant Bit Rate) traffic relative to one ATM (Asynchronous Transfer Mode) connection at $70.67 \mathrm{kbit} / \mathrm{s}$, which corresponds to a cell interarrival time of $6 \mathrm{~ms}$. The application envisioned with this scenario is to provide wireless access to an ATM based data acquisition and control system [11].

Table 1 presents the values of the configurable parameters of the network that were used in the simulations. For the fixed parameters, please refer to the standards.

Table 1. HIPERLAN/2 parameters

\begin{tabular}{|l|l|}
\hline LCH transmission rate & $18 \mathrm{Mbit} / \mathrm{s}$ \\
\hline SCH transmission rate & $6 \mathrm{Mbit} / \mathrm{s}$ \\
\hline Uplink / RCH guard time & $2 \mu \mathrm{s}$ \\
\hline Uplink / RCH preamble & $16 \mu \mathrm{s}$ \\
\hline Turn around time & $6 \mu \mathrm{s}$ \\
\hline Window size & 512 \\
\hline
\end{tabular}

The LCH transport channels required for error free transmissions were reserved using the Fixed Capacity Agreement (FCA) procedure. Additional channels required for retransmissions are granted by the proposed scheduling mechanism. In order to minimize the jitter, one $\mathrm{LCH}$ channel is granted for each connection every one out of 3 HIPERLAN/2 frames, i.e., $6 \mathrm{~ms}$. In order to distribute the load among the frames, 10 out of the 30 connections are served on each frame.

The following results are relative to the first connection. The other 29 connections present similar results. Figure 5 displays the complementary cumulative density function (CCDF) of the delay for different values of the bit error rate (BER) seen by the DLC layer, with constant BER. When BER $=0$, the delay variation is almost zero because the cell interarrival time is equal to the FCA interval and there 
are no retransmissions. In this case, the measured channel utilization regarding the 30 connections is 26.9 $\%$.

With channel errors, it can be seen that the proposed scheduling mechanism forces the retransmissions to occur on consecutive frames, helping to keep the delay within acceptable levels when the BER is moderate. However, with $\mathrm{BER}=10^{-3}$, the number of retransmissions is significant, impacting not only the delay, but also the channel utilization, which rises by $80.3 \%$ when compared to the error free case, whereas with $\mathrm{BER}=10^{-4}$ it rises only by $4.8 \%$.

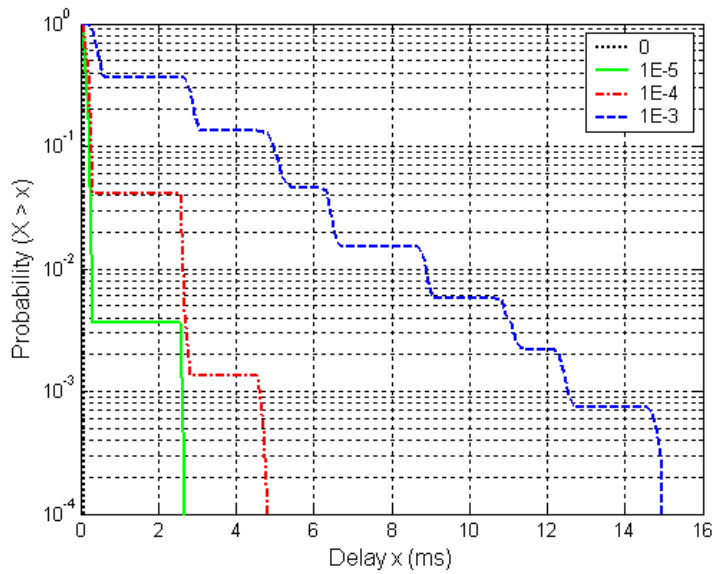

Figure 4. CCDF of the delay with the fast scheduling mechanism and constant BER

Given the varying nature of the wireless channel, more realistic results can be achieved with the GilbertElliot (GE) model. The parameters used in this paper for this model are: $B E R_{\text {bad }}=10^{-3}, B E R_{\text {good }}=0$, $\bar{T}_{\text {bad }}=33 \mathrm{~ms}$ and $\bar{T}_{\text {good }}=100 \mathrm{~ms}$.

Figure 5 compares the delay performance obtained using a constant BER of $10^{-3}$, the Gilbert-Elliot model, and a constant BER of $2.48 \times 10^{-4}$, which corresponds to the average BER of the GE model. The results show that the delay variation with the GE model is larger than that of a model with a constant BER, for the same average BER.

Figure 6 presents the delay performance achieved with the adaptive rate scheme using the Gilbert-Elliot model, and compares it with the use of a fixed transmission rate. The use of mode 4 (18 Mbit/s) all the time results in the largest delay, while the use of mode 1 (6 Mbit/s) results in the lowest delay. However, the channel utilization is significantly higher in this case ( $51 \%$, whereas with mode 4 is $31.5 \%$ ). On the other side, the delay with the proposed adaptive rate scheme is slightly larger than with mode 1 , but the channel utilization is much smaller (32\%).

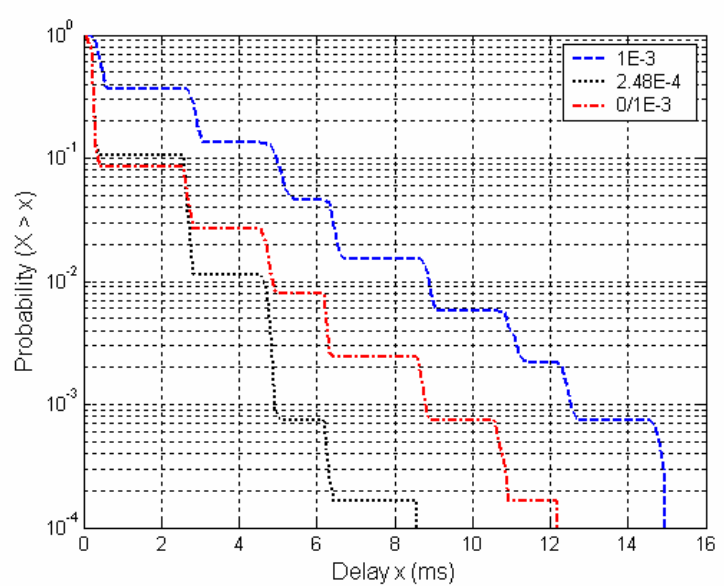

Figure 5. CCDF of the delay with the fast scheduling mechanism and constant or variable BER

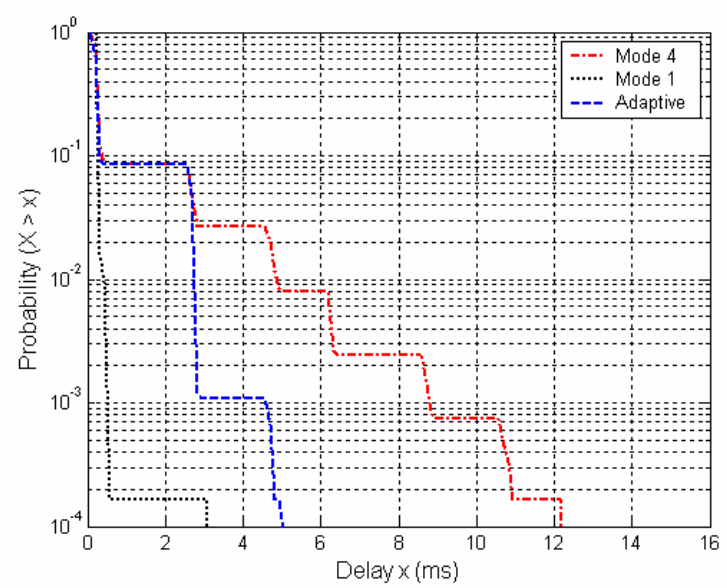

Figure 6. CCDF of the delay with and without the adaptive rate scheme and variable BER

\section{Conclusions}

In this paper we proposed a fast retransmission scheduling mechanism for reliable and timely delivery of real-time traffic in HIPERLAN/2 systems. This mechanism schedules not only the data packets but also the control packets, taking into account the characteristics of the MAC and error control functions of the standard. With this mechanism, retransmissions occur on consecutive frames. Together with the proposed adaptive rate scheme, it helps to maintain the delay under control, allowing the effective use of the HIPERLAN/2 ARQ scheme with real-time traffic.

\section{References}

[1] S. Keshav, An Engineering Approach to Computer Networking, Addison-Wesley, 1999. 
[2] S. Lu et al, "Fair Scheduling in Wireless Packet Networks", IEEE/ACM Transactions on Networking, vol. 7, no. 4, pp. 473-489, 1999.

[3] P. Lin et al, "A Wireless Fair Scheduling Algorithm for Error-Prone Wireless Channels", ACM International Workshop on Wireless Mobile Multimedia WoWMoM 2000, Boston, USA, pp. 11-20, August 2000.

[4] H. Li, G. Malmgren and M. Pauli, "Performance Comparison of the Radio Link Protocols of IEEE802.11a and HIPERLAN/2", IEEE Vehicular Technology Conference VTC2000, Boston, USA, September 2000.

[5] A. Kadelka, A. Hettich, and S. Dick, "Performance Evaluation of the MAC protocol of the ETSI BRAN HIPERLAN/2 standard", European Wireless'99, Munich, Germany, pp. 157-162, October 1999.

[6] A. Doufexi et al, "A Comparison of the HIPERLAN/2 and IEEE 802.11a Wireless LAN Standards", IEEE Communications Magazine, pp. 172-180, May 2002.
[7] J. A. Afonso and J. E. Neves, "Scheduling of real-time traffic in IEEE 802.11 networks", European Conference on the Use of Modern Information and Communication Technologies, Ghent, Belgium, pp. 113-120, April 2004.

[8] ETSI TR 101683 V1.1.1, "Broadband Radio Access Networks (BRAN); HIPERLAN Type 2; System Overview", 2000.

[9] ETSI TS 101 761-1 V1.2.1, "Broadband Radio Access Networks (BRAN); HIPERLAN Type 2; Data Link Control (DLC) Layer; Part 1: Basic Data Transport Functions", 2000.

[10] A. Varga, "OMNET++ Discrete Event Simulation System", User Manual, Technical University of Budapest, Department of Telecommunications, 2000. Available at http://www.omnetpp.org/

[11] J. E, Neves, "Modular Architecture for High Flexibility ATM Based Control System", Asia-Pacific Conference on Communications APCC'99, Beijing, China, 1999. 